\title{
CONTROLLER ORDER REDUCTION WITH GUARANTEED STABILITY AND PERFORMANCE
}

\section{Kathryn E. Lenz, Pramod P. Khargoneter, and John C. Doyle}

University of Minnesota, Honeywell Systems and Research Center, and California Institute of Technology.

\section{INTRODUCTION}

In this paper we consider the problem of controller order reduction for control design for robust performance. In practical control design it may be important to have low order controllers. For example, one may wam to gain schedule a series of LTI (linear, time invariant) controllers, or give simple physical interpretations to the control dymamics. When solving practical design problems using, say, $\mathrm{H}_{\text {. }}$ software it is common to produce controlless of bigh order - equal to the sum of the order of the plant plus each of the weighting fumctions. However, there may be lower order controllers which stabilize the plant and provide satisfactory $\mathrm{H}_{\text {a }}$ closed loop performance. The objectives of a method for controller onder reduction within the $\mathrm{H}_{\mathrm{o}}$ framewort, then, should be to find low order controllers which stabilize a given plant and provides satisfac-

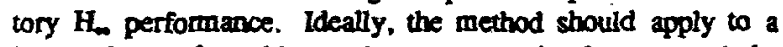
large class of problems, be easy to implement and be guaranteed to work.

The problem of controller onder reduction has been addressed by several authors. We refex the reader to the reference list given in [1] for altemative approaches to controller order recuction. Some approaches focus on preserving closed loop performance with no guarantees on closed loop stability. Other methads preserve closed loop stability but not performance.

We address the problem of maintaining closed loop stability and performance simultaneously. Our method is based on weighted $L$ model reduction of the nominal controller. Since no technique exists for this optimal model reduction problem. we use Hankel nom model recuction techniques $[3],[4]$ to obtain suboptimal controllers.

\section{PROBLEM SETUP}

We consider the general $H_{\text {o }}$ LTI multiple input multiple output control problem. In this setup we have a generalized plant which includes the linear model for the physical system to be controlled, along with weighting functions representing the frequency characteristics of exogenous signals, plant uncertainty, and desired closed loop performance [2].

The generalized plant $P$ can be considered to be composed of four transfer functions $P_{i j} i, j=1,2$ as stown in figure 1: $P_{12}$ is the transfer function from exogenous inpuis to controlled outputs, $P_{22}$ from control inputs to measured outputs, $P_{12}$ from control imputs to controlled outputs, and $P_{21}$ from exogerous inputs to measured outputs. We make the standard assumption that $P_{12}$ is left-invertible and $P_{21}$ is right-invertible at all frequencies.

For a controller $K$, the closed loop transfer function is given by the linear fractional map

\[ F_{1}(P, K)=P_{11}+P_{12} K\left(I-P_{22} K\right)^{-1} P_{21} \]
associated with the matrix $P=\left[\begin{array}{ll}P_{11} & P_{12} \\ P_{21} & P_{22}\end{array}\right]$. Such a map is a
disk to disk transformation from $R L_{-}$to $R_{-m}$.
Suppose $K_{0}$ is a given trigh order controller such that FF $\left(P, K_{0}\right)$ H. We derive weighting functions $W_{1}$ and $W_{2}$ such that if $\left(K_{0}-\hat{K}\right)$ is stable and the weighted $L_{-100 m}$ error betwen $K_{0}$ and $\hat{k}$ is smaller than a specified bound $\bar{\beta}^{-1}, k$ will stabilize $P$ and provide a cloeed loop $L_{L}$ perfomance level of at most $\bar{\beta}$. In other words, we find $W_{1}$ and $W_{2}$ such the if $\left\|W_{1}^{-1}\left[R_{0}-\hat{K}\right] W_{2}^{-1}\right\|_{0} \leq \bar{B}^{-1}$ then $F_{1}(P, \hat{K})$ is stable and $\left\|F_{R}(\mathbf{P}, \hat{R})\right\|_{0} \leq \overline{\mathbf{P}}$.

\section{APPROACH}

In this section we pose the controller order recuction problem as a 2-block perturbation problem similar in spirit to that of the $\mu$ framewot [2]. The standard $\mu$ methods provide exact answers to questions of prorst-case performance for a given set of systems described in terms of linear fractional transfonnations on structured nom bounded perturbations. We assume that a possibly high oniter LTI feedback controller $K_{0} \in R L_{\text {w }}$ has been designed for the generalized plant $P$. The nominal controller $K_{0}$ stabilizes $P$ and $F_{1}\left(P_{1} K_{0}\right){ }_{1}<1$. Here we want to describe all $K$ such that $K$ sabilizes $P$ and $\left\|F_{1}(P, K)\right\|_{\text {Lo }} \leq \bar{\beta}$. It tums out that our technique does not produce the entire set of desirable controllers, but does produce low order controllers with $\beta \leq \sqrt{2}$. Thus, the cost of controller reduction in this scheme is performance degradation by a factor of $\sqrt{2}$.

If $\hat{R}$ is another controller which also stabilizes $P$ then we can always write $R=\left(K_{0}+\hat{\Delta}\right)$ for some real rational $\hat{\Delta}$. The block diagram representation of the closed loop system with controller $\hat{\mathrm{K}}$ can be expressed in terms of the closed loop system with the nominal controller $K_{0}$ and an additive error $\hat{\Delta}$ as shown in figure 1.

This system can then be written as a feedback comection (generalized plant) $\mathbf{P}$ connected with $\hat{\Delta}$ as indicated in figure 1 .

$$
\text { Here } \quad \begin{aligned}
\tilde{\mathbf{P}}_{11} & =\mathbf{P}_{11}+\mathbf{P}_{12} \mathbf{K}_{0}\left(I-\mathbf{P}_{22} K_{0}\right)^{-1} \mathbf{P}_{21} \\
\tilde{\mathbf{P}}_{12} & =\mathbf{P}_{12}\left(I-\mathbf{P}_{22} K_{0}\right)^{-1} \\
\tilde{\mathbf{P}}_{21} & =\mathbf{P}_{21}\left(I-\mathbf{P}_{22} K_{0}\right)^{-1} \\
\tilde{\mathbf{P}}_{22} & =\left(I-P_{22} K_{0}\right)^{-1} \mathbf{P}_{22}
\end{aligned}
$$

Since $K_{0}$ internally stabilizes $P, P_{i j}$ is stable for $i j=1,2$.

Let $\bar{D}=\left\{\left[\begin{array}{ll}1 & 0 \\ 0 & \lambda\end{array}\right]: \lambda \in R^{+}\right\}$. Define, for $M$ a corstant complex matrix, $\mu(M):=\inf _{D \in \bar{D}} \sigma\left(D M D^{-1}\right)$. For a given $\check{P}$, let $\Sigma_{\mathrm{P}}=\left\{\Delta:\|\Delta\|_{0}<1\right.$ and $\left(I-\tilde{P}_{22} \Delta\right)^{-1}$ is bounded $\}$. The 2 -block $\mu$ test [5],[2] then shows that: $\left[F_{1}(\tilde{P}, \Delta) \text {, }\right\}_{0} \leq 1$ for all $\Delta \in \sum_{p}$ if and only if $\sup _{j \omega} \mu(\tilde{P}(j \omega)) \leq 1$.

Assuming that $\sup \mu(\bar{P}(j \omega)) \leq 1$, the 2-block $\mu$ test ensures that if $\| \hat{K}-K_{d} d \hat{j}<1$, then $F_{l}(P, \hat{K})=F_{l}(\tilde{P}, \hat{\Delta})$ will have satisfactory $L_{\infty}$ performance. If in addition we have that $\left\|\tilde{P}_{2}\right\|_{0}<1$, then $F_{1}(\tilde{P}, \Delta)$ is stable for all stable $\Delta \in \sum_{p}$ However, the entire set of perturbations $\Delta$ for which the closed loop system will have satisfactory $L_{\infty}$ performance and be stable can be difficult to determine. It may be that $\hat{\Delta}=\left(\mathrm{K}_{0}-\hat{\mathrm{K}}\right)$ is not in $\Sigma_{p}$ but $\hat{K}$ still provides acceptable performance and closed locp 
stability. In short, a desirable reduced order controller may not correspond to a "worst-case" $\Delta$, or even a stable $\Delta$. Hence, this onder reduction technique is, in general, conservative. However, in a special case it is not conservative.

For a rational matrix $M$ in $L_{-}, M\left(j \omega_{0}\right)$ is called a $\lambda$ iscometry [5] if there is a positive scalar $\lambda$ such that $\left[\begin{array}{cc}M_{11} & \lambda^{-1} M_{12} \\ \lambda M_{21} & M_{22}\end{array}\right]\left(j \omega_{0}\right)$ is an isometry.

Theorem 1: $P\left(j \omega_{0}\right)$ is a $\lambda$-isometry for each $\omega_{0}$ if and only if \|\|$F_{r}(\tilde{P}, \Delta) \|_{\infty} \leq 1$ if and only if $\left.\|\Delta\|_{\infty} \leq 1\right\}$. Further, if $\tilde{P}\left(j \omega_{0}\right)$ is a $\lambda$-isometry for each $\omega_{\infty}, F_{n}(\vec{P}, \Delta)$ is stable for all stable $\Delta \in \Sigma_{\mathbf{p}}$.

In general, $\overline{\mathbf{P}}\left(j \omega_{\alpha}\right)$ will not be a $\lambda$-isometry, and so we will not have an if and only if relationship between closed loop performance and the norm of $\Delta$.

Suppose that $\mu(\tilde{P}(j \omega))=\beta(j \omega)$. Then $\mu\left(\beta(j \omega)^{-1} \tilde{P}(j \omega)\right) \leq 1$ for all $j \omega$. So our 2-block $\mu$ test holds for $\beta^{-1} \hat{P}$. Consider

$$
F_{1}\left(\beta^{-1} \tilde{P}, \Delta\right)=\beta^{-1}\left(\tilde{P}_{11}+\tilde{P}_{12}\left(\beta^{-1} \Delta\right)\left(I-\tilde{P}_{22}\left(\beta^{-1} \Delta\right)\right)^{-1} \tilde{P}_{21}\right) \text {. }
$$

From (2) we have that $\| F_{1}\left(\tilde{P}, \beta^{-1} \Delta \|_{k} \leq \sup _{j \omega} \beta(j \omega):=\overline{\bar{P}}\right.$ for all $\Delta \in \Sigma_{\beta-1}$. Therefore, we can guarantee a level $\bar{\beta}$ of closed loop system performance for all controllers $\hat{R}$ such that $\left\|\beta\left(\mathrm{K}_{0}-\hat{\mathrm{K}}\right)\right\|_{\infty} \leq 1$.

We can use weighting functions as shown in figure 1 to alter the shape of $\tilde{\mathbf{P}}$. We can not in general tum $\tilde{\mathrm{P}}\left(\mathrm{j} \omega_{0}\right)$ into a $\lambda$-isometry with weighting functions located as in figure 1 , but we can make $\overline{\mathrm{P}}\left(j \omega_{2}\right)$ closer to an isometry in some sense.

Lemma 3: $\left[\begin{array}{ll}M_{11} & M_{12} \\ M_{21} & M_{22}\end{array}\right]$ is an isometry if and only if

$$
\begin{gathered}
\mathbf{M}_{.1}:=\left[\begin{array}{l}
\mathbf{M}_{11} \\
\mathbf{M}_{21}
\end{array}\right] \text { and } \mathbf{M}_{2}:=\left[\begin{array}{l}
\mathbf{M}_{12} \\
\mathbf{M}_{22}
\end{array}\right] \text { are isometries } \\
\text { and } \mathbf{M}_{11}^{*} \mathbf{M}_{12}+\mathbf{M}_{21}^{*} \mathbf{M}_{22}=0 .
\end{gathered}
$$

For an arbitrary $\tilde{\mathrm{P}}$, the weights

$$
\begin{aligned}
W_{1} & :=\text { outer }\left[\left(I-P_{11}^{*} P_{11}\right)^{1 / 4} P_{21}^{-1}\right] \\
W_{2} & :=\text { outer }\left[\left(P_{12}^{*} P_{12}+P_{2}^{*} W_{1}^{*} W_{1} P_{22}\right)^{-4 / 2}\right]
\end{aligned}
$$

make $T_{.1}\left(j \omega_{0}\right)$ and $T_{2}\left(j \omega_{0}\right)$ isometries for each $\omega_{\alpha}$ where

$$
\mathrm{T}:=\left[\begin{array}{ll}
\mathrm{T}_{11} & \mathrm{~T}_{12} \\
\mathrm{~T}_{21} & \mathrm{~T}_{22}
\end{array}\right]=\left[\begin{array}{cc}
1 & 0 \\
0 & \mathrm{~W}_{1}
\end{array}\right] \tilde{\mathrm{P}}\left[\begin{array}{cc}
\mathrm{I} & 0 \\
0 & \mathrm{~W}_{2}
\end{array}\right] .
$$

However, $T_{.1}$ and $T_{2}$ will in general not satisfy (5) and hence $\sigma\left(T\left(j \omega_{\alpha}\right)\right) \geq 1$. One can verify that the largest $\delta\left(T\left(j \omega_{0}\right)\right)$ can be is $\sqrt{2}$, and in fact $\mu\left(T\left(j \omega_{\alpha}\right)\right)$ can be as large as $\sqrt{2}$. This can happen only when $T_{.1}\left(j \omega_{\alpha}\right)$ is a scalar multiple of $T_{2}\left(j \omega_{0}\right)$ for some $\omega_{0}$

For the general system shown in figure 1 , we have $\hat{\mathbf{R}}=\mathrm{K}_{0}+\mathrm{W}_{1} \hat{\Delta} \mathbf{W}_{2}$ and so $W_{1}^{-1}\left(\hat{R}-\mathrm{K}_{0}\right) W_{2}^{-1}=\hat{\Delta}$. This defines a weighted model reduction problem: If sup $\mu(T(j \omega))=\bar{\beta}$ and $\left\|W_{1}^{-1}\left(\hat{K}-K_{0}\right) W_{2}^{-1}\right\|_{L}<\bar{\beta}^{-1}$, then $\left\|F_{1}(P, \hat{K})\right\|_{\infty} \leq \bar{\beta} \leq \sqrt{2}$. We bave constructed $T$ so that $T_{i j}$ for $i, j=1,2$ are stable and $\left\|\mathrm{T}_{22}\right\|_{\mathrm{L}}<1$. Therefore, if $\hat{\Delta}$ is a contraction at every frequency and is stable, then $F_{1}(P, R)$ is stable.

Implementation of this method of controller order reduction requires computing reduced order controllers within a weighted $\mathrm{L}_{\infty}$ ball centered at $\mathrm{K}_{0}$ with left radius $\mathrm{W}_{\mathrm{l}}^{-1}$ and right radius $W_{2}^{-1}$. We can ensure that the error between the reduced and original controller is a stable proper $\mathrm{L}_{\text {. }}$ function by performing weighted Hankel norm model reduction of $K_{0}$ [4]. The amount of Hankel-norm error we incur is always less than or equal to the $L_{-}$-norm error. Therefore, we must check $\left\|W_{1}^{-1}\left(K_{0}-\hat{K}\right) W_{2}^{-1}\right\|_{L}<\bar{\beta}^{-1}$ to guarantee closed loop stability and that $\left\|F_{1}(\mathbf{P}, \hat{K})\right\|_{\infty}<\overline{\mathbf{B}}$.

This method is in general conservative. There may be a desirable low order controller whose weighted $\mathrm{L}_{\infty}$-norm error from $K_{o}$, the nominal controller, is greater than $\bar{\beta}^{-1}$. We may not find such a $\hat{\mathbf{K}}$ even if we allow our weighted error to be as large as we like. For example, if the number of unstable poles of $\hat{K}$ is different than the number of unstable poles of $K_{0}$ we will not get $\hat{\mathbf{K}}$ by our proposed weighted madel reduction procedure. A way to generalize our method is to model reduce a coprime factorization of $K_{o}$ instead of $K_{0}$ directly. We can formulate an order reduction scheme for a coprime factorization of $\mathrm{K}_{\mathrm{o}}$ from a 2-block $\mu$ problem analogous to the one considered in section 3 . This allows the number of unstable poles of a reduced order controller $\hat{R}$ to vary. Also, one could choose weighting functions other than $W_{1}$ and $W_{2}$ given in (6), which would lead to different sets of attainable reduced order controllers. Because we use a weighted Hankel-norm approximation to $K_{o}$ we may not get the best weighted $L_{-\infty}-n o r m$ approximations to $\mathrm{K}_{\mathrm{o}}$.

We have also considered approaches which directly use the parametrization of all controllers which stabilize a given plant and provide a specific level of $\mathrm{H}_{\infty}$ performance in terms of a linear fractional transformation of the set of all contractions in $\mathrm{RH}^{\circ}$. However. so far this has not proven useful for controller order reduction.

\section{REFERENCES}

[1] B. D. O. Anderson and Y. Liu "Controller Reduction: Concepts and Approaches", A.C.C. 1987, pp. 1-9.

[2] J. C. Doyle, Lecture Notes, ONR/Honeywell Workshop Advances in Multivariable Control, Oct., 1984, Mpls, MN.

[3] K. Glover, "All optimal Hankel-norm approximations of linear multivariable systems and their L-error bound", Intemat. J. Control, Vol. 39, No. 6, (1984) 1115-1193.

[4] G. A. Latham and B. D. O Anderson, "Frequency-weighted optimal Hankel-nom approximation of stable transfer functions", Systems and Control Letters, Vols, No.4,(1984) 229. 236.

[5] R. M. Redheffer, "On a certain linear fractional transformation", Jownal Math. Physics, 39 (1960), 269-286.

This research was supported in part by the National Science Foundation under Grant No. ECS-8451519, grants from Honeywell, General Electric Co., and ONR Research Grant N00014-82-C-0157.

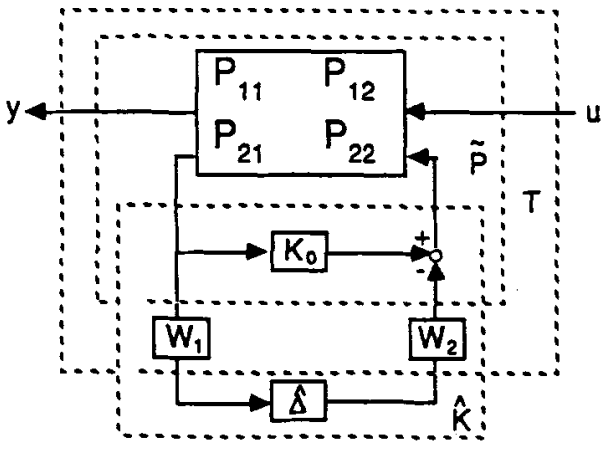

Figure 1. General System with Perturbed Controller 